\title{
Squalamine and its Derivatives as Potential Antitubercular Compounds
}

\section{Correspondance}

To the Editor,

Squalamine is an aminosterol isolated from the dogfish shark Squalas acanthias and identified for its antimicrobial activity. ${ }^{1}$ It also inhibits the growth of blood vessels within solid tumors and has been widely investigated as an antitumor agent, and has been included in clinical trials. ${ }^{2}$ Squalamine has been shown to possess improved antitumor activity when co-administered with carboplatin or cisplatin. ${ }^{3}$ Recently, its potential therapeutic spectrum has been widened by the discovery of broad-spectrum systemic antiviral activity. ${ }^{4}$ We believe squalamine itself or squalamine derivatives are a potential class of targeted antitubercular agents. The hypothesis that these derivatives might work in this way is based on two characteristics of Mycobacterium tuberculosis (MTB): the waxy, hydrophobic barrier provided by mycolic acids on the mycobacterial surface, and the affinity of mycobacteria for cholesterol. There are both steroidal and amphiphilic compounds have antimycobacterial activity and may be able to permeate the fatty acid layers of this structure because of their non-polar carbon based skeleton. ${ }^{5}$ Furthermore, lipophilic fatty acid derivatives have been shown to be active against several strains of mycobacteria, including ones that were multidrug resistant (MDR). ${ }^{6}$ One critical protein that is contained by MTB during macrophage invasion is the tryptophane aspartate-containing coat protein (TACO). ${ }^{7}$ Mycobacteria actively recruit and retain TACO to prevent fusion with lysosomes. After phagocytosis, macrophages are normally converted to lysosomes, which degrade consumed material, but mycobacteria retain TACO at the phagosome stage, which prevent conversion to 
lysosomes. ${ }^{8}$ This absence of lysosomes helps ensure the survival of MTB within the macrophage. Importantly from a drug targeting standpoint, TACO associates with the phagosome in a cholesterol dependent manner. Cholesterol inhibition reduces macrophage uptake of $M$. bovis by $85-90 \%$ and MTB by $85 \%$, and mycobacteria display an intrinsic high binding capacity for cholesterol. ${ }^{7}$ The affinity of mycobacteria for cholesterol could be exploited by designing drugs that have structural similarities to cholesterol. Previously, we have synthesized neomycincholesterol hybrids in an attempt to engineer this uptake mechanism. ${ }^{5}$

The mechanism for the antimicrobial activity of squalamine has been widely studied and initially it was believed to act as a proton ionophore. The spermidine side chain (Figure 1) could interact with the 24 -sulfate, forming a polar face that would line the aqueous interior of a transmembrane pore. The formation of this stable pore would compromise the integrity of the organism, resulting in cell-death. Other groups have synthesized analogs with shorter amine chains or hydroxyl groups at the 3position that are incapable of forming a membrane pore and these also possess antimicrobial activity. Structure-activity relationships have shown that different analogs have quite varied potencies against different organisms. ${ }^{9}$ These fluctuations in antimicrobial activity suggest that these agents are acting upon specific sites in each organism that differs for each organism. Another study showed that squalamine is not a proton ionophore ${ }^{10}$ and recent work indicates its antibacterial action results from membrane depolarization. ${ }^{11}$ This information leads us to believe that the 24 sulfate may not be necessary for antimicrobial activity. ${ }^{12}$ Thus, we initially targeted compounds $\mathbf{1}$ and $\mathbf{2}$ as our model squalamine analogs to test their physicochemical properties (Figure 1). 

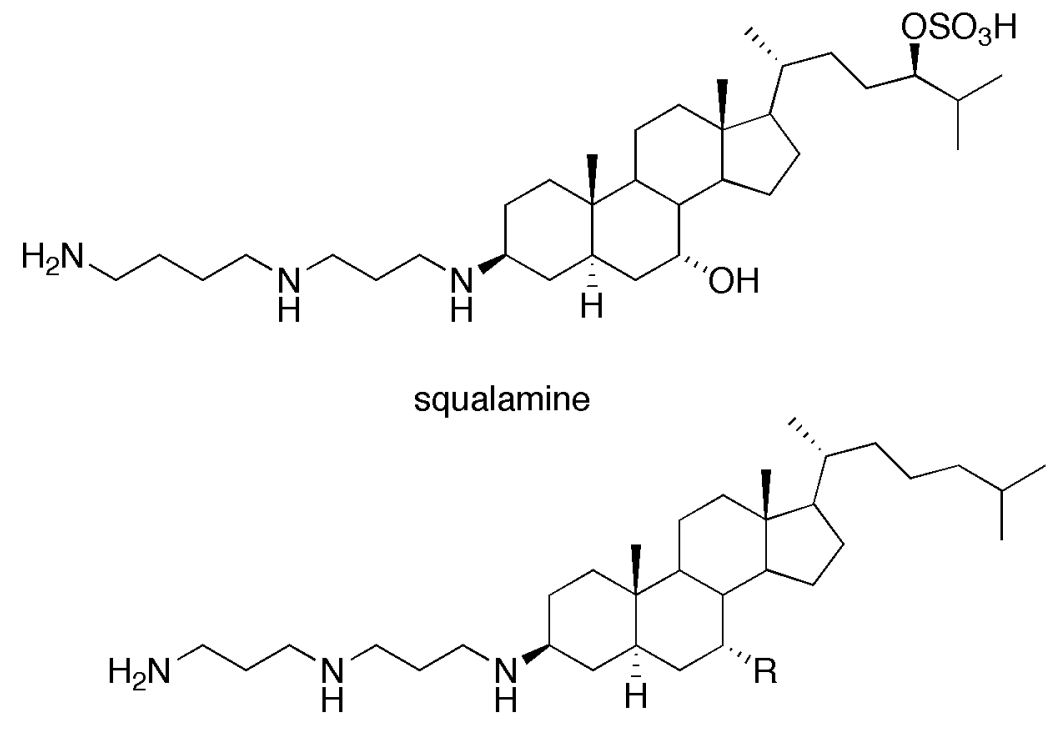

$$
\begin{aligned}
& \text { 1: } \mathrm{R}=\mathrm{H} \\
& \text { 2: } \mathrm{R}=\mathrm{OH}
\end{aligned}
$$

Figure 1. Squalamine structure and target compounds

Compound 1 was synthesized in a single step by reductive amination of cholestenone with the requisite triamine. ${ }^{13}$ However, initial attempts to dissolve or formulate this compound for antimycobacterial studies proved difficult. In the electrospray mass spectra of this and related compounds, dimers and trimers were often observed. The amphiphilic nature of this compound may require coadministration of a cyclodextrin or some other solubilizing agent to disrupt aggregation. The tendancy toward self-aggregation by $\mathbf{1}$ may be diminished when an axial hydroxy group is added at C-7 (e.g.-2) as is present in squalamine itself. This axial alcohol is also present in the natural surfactant cholic acid (albeit with a differing configuration at the $\mathrm{AB}$ bridgehead C-5). In fact, a patent on the 3-epimer of this compound and close derivatives of squalamine has recently been filed for use as antidiabetic agents indicative of improved physicochemical properties for $\mathbf{2}$ relative to $\mathbf{1}^{14}$ Although we have been unable to obtain antimycobacterial data on these compounds, due to the desperate need for new TB drugs, ${ }^{15}$ we would encourage those 
who have access to the natural product to have it tested against MDR-TB as we are not aware of any such study.

\section{References}

[1] K. S. Moore, S. Wehrli, H. Roder, M. Rodgers, J. M. Forrest, D. McCrimmon, and M. Zasloff, "Squalamine - An Aminosterol Antibiotic from the Shark," PNAS, 1993, 90, 1354-58. For a recent review, see: K. Alhanout, J. M. Rolain, and J. M. Brunel, "Squalamine as an Example of a New Potent Antimicrobial Agents Class: A Critical Review," Curr. Med. Chem. 2010, 17, 3909-17.

[2] P. Bhargava, J. L. Marshall, W. Dahut, N. Rizvi, N. Trocky, J. I. Williams, H. Hait, S. Song, K. J. Holroyd, and M. J. Hawkins, "A phase I and pharmacokinetic study of squalamine, a novel antiangiogenic agent, in patients with advanced cancers," Clin. Cancer Res. 2001, 7, 3912-19.

[3] D. Li, J. I. Williams, and R. J. Pietras, "Squalamine and cisplatin block angiogenesis and growth of human ovarian cancer cells with or without HER-2 gene overexpression," Oncogene 2002, 21, 2805-14.

[4] M. Zasloff, A. P. Adams, et al. "Squalamine as a broad-spectrum systemic antiviral agent with therapeutic potential" PNAS 2011, 108, 15978-83.

[5] S. Quader, S. E. Boyd, I. D. Jenkins, and T. A. Houston, "Multisite Modification of Neomycin B: A Combined Mitsunobu and Click Chemistry Approach" J. Org. Chem. 2007, 72, 1962-79.

[6] N. M. Parrish, T. Houston, P. B. Jones, C. A. Townsend, and J. D. Dick, "In Vitro Activity of a Novel Antimycobacterial Compound, $N$-Octanesulfonylacetamide, and its Effect on Lipid and Mycolic Acid Synthesis," Antimicrob. Agents Chemother. 2001, 45, 1143-50.

[7] J. Gatfield and J. Pieters, "Essential Role for Cholesterol in Entry of Mycobacteria into Macrophages," Science 2000, 288, 1647-1650.

[8] J. Pieters, "Entry and survival of pathogenic mycobacteria in macrophages," Microbes and Infection 2001, 3, 249-255.

[9] S. R. Jones, W. A. Kinney, X. Zhang, L. Jones, and B. S. Selinsky, "Synthesis and characterization of analogs of the antimicrobial compound squalamine," Steroids 1996, 61, 565-571.

[10] B. S. Selinsky, R. Smith, A. Frangiosi, B. Vonbaur, and L. Pedersen, "Squalamine is not a proton ionophore," Biochim. Biophys. Acta 2000, 1461, 135141 ;

[11] K. Alhanout, S. Malesinki, N. Vidal, V. Peyrot, J. M. Rolain, and J. M. Brunel, "New insights into the antibacterial mechanism of action of squalamine" $J$. Antimicrob. Agents Chemother, 2010, 65, 1688-1693. 
[12] Y. Shu, S. R. Jones, W. A. Kinney, and B. S. Selinsky, "The synthesis of spermine analogs of the shark aminosterol squalamine," Steroids 2002, 67, 291-304.

[13] We used excess dipropylenetriamine (6 equiv.) and $\mathrm{NaBH}_{3} \mathrm{CN}$ (5 equiv.) relative to cholestane, but other methods have been developed: C. Salmi, C. Loncle, N. Vidal, Y. Letourneux, and J. M. Brunel, "New stereoselective titanium reductive amination synthesis of 3-amino and polyaminosterol derivatives possessing antimicrobial activities," Eur. J. Med. Chem. 2008, 43, 540-547.

[14] M. McLane, I. Ruiz-White, and H. Wolfe, "Use of steroid compounds inhibiting protein tyrosine phosphatases for treating diabetes," PCT Int. Appl. 2009, WO 200903232.

[15] P. J. Brennan , "Approaches to developing new drugs against tuberculosis," Tuberculosis 2009, 89, 329-330.

Brian T. Walker ${ }^{\mathrm{a}}$, Todd A. Houston* ${ }^{\mathrm{a}, \mathrm{b}}$

${ }^{a}$ Department of Chemistry, Virginia Commonwealth University, Richmond, VA, 23284-9059, USA

' Institute for Glycomics, Gold Coast Campus, Griffith University, QLD 4222, Australia and School of Biomolecular and Physical Sciences, Nathan Campus, Griffith University, QLD 4111, Australia

Correspondence should be addressed to Todd A. Houston, T.Houston@griffith.edu.au 